\title{
Hybrid Precoding for MISO Broadcasting SWIPT Systems: A Stochastic Optimization Approach
}

\author{
Konstantinos Ntougias*, Ioannis Krikidis*, Georgios K. Papageorgiou ${ }^{\dagger}$ and Mathini Sellathurai ${ }^{\dagger}$ \\ *Department of Electrical and Computer Engineering, University of Cyprus, Nicosia, Cyprus \\ ${ }^{\dagger}$ School of Engineering and Physical Sciences, Heriot-Watt University, Edinburgh, UK \\ Email: \{ntougias.konstantinos, krikidis\}@ucy.ac.cy, \{g.papageorgiou, m.sellathurai\}@hw.ac.uk
}

\begin{abstract}
This paper investigates the hybrid precoding (HP) design for simultaneous wireless information and power transfer in a multiple-input single-output broadcast channel setup where the terminals adopt the power splitting architecture. The problem of interest is the maximization of the signal-to-interference-plusnoise-ratio and the harvested power for all terminals under a total transmit power constraint. Our focus is on the derivation of frequency- and setup-agnostic low-complexity HP methods. Two baseline approaches for the determination of the analog precoder are considered. In the first one, the phases are computed via the singular value decomposition (SVD) of the channel matrix, while in the second they are selected randomly. Then, the baseband precoder is computed by applying semidefinite relaxation (SDR) to the problem under study. Alternatively, we combine the aforementioned analog precoders with a fixed zero-forcing baseband precoder, in order to further reduce the computational load. Another proposed strategy focuses on the minimization of the Euclidean distance between the optimal fully-digital precoder, which is obtained via SDR, and the hybrid one. To this end, an alternating minimization algorithm that employs Gaussian smoothing to convexify the problem and utilizes stochastic gradient descent to update the phases is introduced. The performance of the proposed HP methods is comparatively evaluated versus the one achieved by the optimal fully-digital precoder via numerical simulations. The simulation results indicate that the stochastic optimization approach presents a favorable performance-complexity trade-off as well as substantial power gains.
\end{abstract}

Index Terms-Simultaneous wireless information and power transfer (SWIPT), MISO broadcast channel, hybrid precoding, stochastic optimization, Gaussian smoothing.

\section{INTRODUCTION}

A massive growth of low-power communication devices, such as smartphones, wireless sensors, and Internet-of-Things (IoT) nodes, is anticipated in the foreseeable future [1]. Simultaneous wireless information and power transfer (SWIPT) is considered a promising approach for prolonging the battery lifetime of such energy-constrained devices, whilst ensuring a prescribed quality-of-service (QoS) level [2]. This is achieved by exploiting the power of the information-bearing signals that are transmitted in the downlink for energy harvesting (EH) purposes. The realization of SWIPT systems requires the design of novel transmission strategies [3]-[7].

Our focus in this paper is on multi-user multiple-input multiple-output (MU-MIMO) SWIPT setups. It is well-known that the performance of MU-MIMO systems improves, from a sum-rate perspective, with the number of transmit antennas.

978-1-7281-4490-0/20/\$31.00 (c) 2020 IEEE
Under a fully-digital transmitter architecture, though, where each antenna element is fed by a radio frequency (RF) chain, even the installation of a moderate number of antennas leads to substantial power consumption. This is problematic for small access points (AP) in IoT setups and small-cell base stations or remote radio units in cellular mobile radio communication networks, which commonly have limited power supply. Furthermore, in view of the ongoing ultra-densification of the radio access network infrastructure, this paradigm raises significant cost considerations. Analog-digital (A/D) transmitters provide a workaround to the above problems by reducing the number of RF units required for approaching a target performance level. This is accomplished by combining a low-dimensional digital or baseband (BB) precoder with a high-dimensional analog or $\mathrm{RF}$ one. The latter is commonly implemented with the help of phase shifters [8].

The joint optimization of the RF and $\mathrm{BB}$ precoders is a non-trivial task, due to the non-convex constant modulus constraints imposed by the phase shifters and the coupling between these precoders. Massive MIMO (mMIMO) literature is rich on corresponding hybrid precoding (HP) techniques, which typically decouple the optimization of these precoders. While some methods exploit the sparsity of millimeter-wave (mmWave) channels [9], others are frequency-agnostic. However, these schemes present high computational complexity. For instance, the matrix decomposition (MDP) method presented in [10] involves as many quadratic programming tasks as the number of antennas, whereas the conjugate gradient method described in [11] makes use of backtracking line search. Similarly, the Nelder-Mead simplex scheme applied in [12] performs multiple matrix inverse or associated $\mathrm{QR}$ factorization recomputations.

Interestingly, the application of HP in SWIPT systems has been studied mainly in the contexts of multi-group multicasting [13] and mmWave mMIMO communication [14], [15]. The only relevant work that focuses on sub- $6 \mathrm{GHz}$ spectrum and conventional (non-massive) MIMO broadcasting is [16], to the best of our knowledge. This study considers the total transmit power (TTP) minimization problem for a multiple-input singleoutput broadcast channel (MISO BC) with a single information receiver and multiple energy receivers under $\mathrm{QoS}$ and $\mathrm{EH}$ constraints. To this end, the authors introduce a HP method that is based on the setup-specific geometric representation of the optimization problem under study. 
In this work, we extend the study on SWIPT with energyefficient transmitters by investigating the design of setup- and frequency-agnostic low-complexity HP schemes. We consider a MISO BC setup with terminals that adopt the power splitting (PS) architecture to concurrently perform information decoding (ID) and EH and focus on the signal-to-interference-plusnoise-ratio (SINR) / EH balancing problem, which is closely related to the TTP minimization problem. Two baseline RF precoder design approaches are described. In the first one, phase computation is based on the singular value decomposition (SVD) of the channel matrix. The second technique refers to random phase selection. After fixing the RF precoder, we can either obtain the BB precoder by applying the semi-definite relaxation (SDR) framework or we can design it according to the zero-forcing $(\mathrm{ZF})$ principle. Next, we describe a stochastic optimization approach based on an iterative minimization algorithm that alternates between the RF and $\mathrm{BB}$ precoder updates. The goal of this algorithm is to minimize the Euclidean distance between the optimal fully-digital precoder and the hybrid one. The former is computed via SDR. In this case, Gaussian smoothing is applied to the multi-extremal cost function, in order to convexify it. Then, the stochastic gradient descent (SGD) algorithm is used to determine the phases. By generating a sequence of minimization runs for the smoothed cost function with reduced amount of smoothing in each one, we filter out the approximation noise, thus enabling SGD to gradually converge towards its global minimum. Numerical simulations indicate that the proposed stochastic optimization approach approaches the performance of the optimal fully-digital solution and presents substantial power savings. In addition, they shed light on the effect of various parameters on the performance of these HP methods.

The remainder of the paper is organized as follows: In Section II, the system model and the problem formulation are introduced. Section III and Section IV present the proposed HP methods and fixed HP schemes, respectively. Performance evaluation via numerical simulation results is conducted in Section V. Section VI provides our conclusions.

Notation: $a$, a, and $\mathbf{A}$ denote a scalar, a column vector, and a matrix, respectively. $\mathbb{R}, \mathbb{C}$, and $\mathbb{N}$ denote the sets of real, complex, and natural numbers, respectively. $j \triangleq \sqrt{-1}$ and $\operatorname{Re}\{\cdot\}$ denote the imaginary unit and the real part of a complex scalar, respectively. $|\cdot|$ refers to the magnitude of a complex scalar. $\mathbb{C}^{n}$ and $\mathbb{C}^{m \times n}$ represent the sets of complex $n$-dimensional vectors and $m \times n$ matrices, respectively. $[\mathbf{a}]_{n}$ and $[\mathbf{A}]_{m, n}$ denote the $n$-th element of a and the $(m, n)$ entry of $\mathbf{A}$, respectively. $\mathbf{I}_{n}$, $\mathbf{0}_{m \times n}$, and $\mathbf{0}_{n}$ denote the $n \times n$ identity matrix, $m \times n$ null matrix, and $n$-dimensional null vector, respectively. rank $(\cdot)$, $\operatorname{tr}(\cdot)$, and $\operatorname{det}(\cdot)$ correspond to the rank, trace, and determinant of a matrix, respectively, whereas $\operatorname{diag}(\cdot)$ denotes the conversion of a vector into a diagonal matrix. $(\cdot)^{*},(\cdot)^{T},(\cdot)^{\dagger},(\cdot)^{-1}$, $(\cdot)^{\#},\|\cdot\|$, and $\|\cdot\|_{F}$ denote the conjugate, transpose, conjugate transpose, inverse, Moore-Penrose pseudo-inverse, Euclidean $\left(L_{2}\right)$ norm, and Frobenius norm of a matrix, respectively. vec $(\cdot)$ represents the vectorization of a matrix, while $\otimes$ and $\odot$ denote the Kronecker and Handamard (element-wise) matrix product operations, respectively. $\mathbf{A} \succeq 0$ indicates that $\mathbf{A}$ is a positive semi-definite matrix. A circularly symmetric complex Gaussian (CSCG) random variable $z$ with mean $\mu$ and variance $\sigma^{2}$ is denoted as $z \sim \mathcal{C N}\left(\mu, \sigma^{2}\right)$. By $\mathbf{X} \sim \mathcal{M N}_{m \times n}(\mathbf{M}, \boldsymbol{\Sigma}, \mathbf{\Psi})$ we denote that the random matrix $\mathbf{X} \in \mathbb{C}^{m \times n}$ follows the matrix Gaussian distribution with mean $\mathbf{M} \in \mathbb{C}^{m \times n}$ and covariance matrices $\boldsymbol{\Sigma} \in \mathbb{C}^{m \times m}$ and $\boldsymbol{\Psi} \in \mathbb{C}^{n \times n}$. $\mathbb{E}[\cdot]$ represents the expectation operator and $\mathcal{U}(\cdot)$ denotes the uniform distribution. $\nabla f$ denotes the gradient of function $f$, whereas $f * g$ denotes the convolution of $f$ with $g$.

\section{SyStem Model AND PROBlem Formulation}

\section{A. Fully-Digital Transmitter}

We consider a MISO BC for SWIPT consisting of a fullydigital AP with $N_{t}$ antennas (and RF chains) and $K \leq N_{t}$ single-antenna user equipment (UE) devices that employ singleuser detection. The AP applies linear precoding at the digital $\mathrm{BB}$ to serve the users on a single time-frequency resource. Availability of perfect channel state information (CSI) at both the AP and the terminals is considered. The latter devices utilize PS, i.e., a portion $\rho_{k} \in(0,1)$ of the received signal's power at the $k$-th $\mathrm{UE}(k \in \mathcal{K}=\{1, \ldots, K\})$ is driven to the information decoder for $\mathrm{BB}$ conversion and data detection whereas the remaining portion is driven to the energy harvester for conversion into a direct current (DC) voltage and energy storage, as illustrated in Fig. 1.

1) System Model: Due to the limited communication range in practical SWIPT systems, there is typically a dominant lineof-sight (LoS) path between the AP and each UE, in addition to the multiple non-LoS (NLoS) paths attributed to the reflection and scattering of the propagating radio signal by obstacles in the environment. Hence, we consider quasi-static frequency-flat uncorrelated Rician fading channels. That is, the complex BB channel between the $k$-th UE and the AP, denoted as $\mathbf{h}_{k} \in \mathbb{C}^{N_{t}}$, is expressed as

$$
\mathbf{h}_{k}=\sqrt{\frac{R}{R+1} L\left(d_{k}\right)} \mathbf{h}_{k}^{\mathrm{LoS}}+\sqrt{\frac{1}{R+1} L\left(d_{k}\right)} \mathbf{h}_{k}^{\mathrm{NLoS}}, k \in \mathcal{K},
$$

where $R$ is the Rician factor, $L\left(d_{k}\right)$ corresponds to the distancedependent path loss at propagating distance $d_{k}, \mathbf{h}_{k}^{\mathrm{LoS}} \in \mathbb{C}^{N_{t}}$ refers to the LoS (specular) component, and $\mathbf{h}_{k}^{\text {NLoS }} \in \mathbb{C}^{N_{t}}$ represents the Rayleigh fading component. The path loss is given by

$$
L\left(d_{k}\right)=C_{0}\left(\frac{d_{k}}{d_{0}}\right)^{-\alpha}
$$

where $C_{0}$ is the path loss at a reference distance $d_{0}=1 \mathrm{~m}$ in the far-field of the AP and $\alpha$ denotes the path loss exponent. By assuming a uniform linear array (ULA) with omni-directional transmit antennas, the LoS component is given by

$$
\mathbf{h}_{k}^{\mathrm{LoS}}=\left[\begin{array}{lll}
\alpha_{0} & \cdots & \alpha_{N_{t}-1}
\end{array}\right]^{T}, \quad k \in \mathcal{K},
$$

where

$$
\alpha_{m-1}=e^{-j(m-1)(2 \pi d / \lambda) \sin \left(\varphi_{k}\right)}, m \in \mathcal{M},
$$




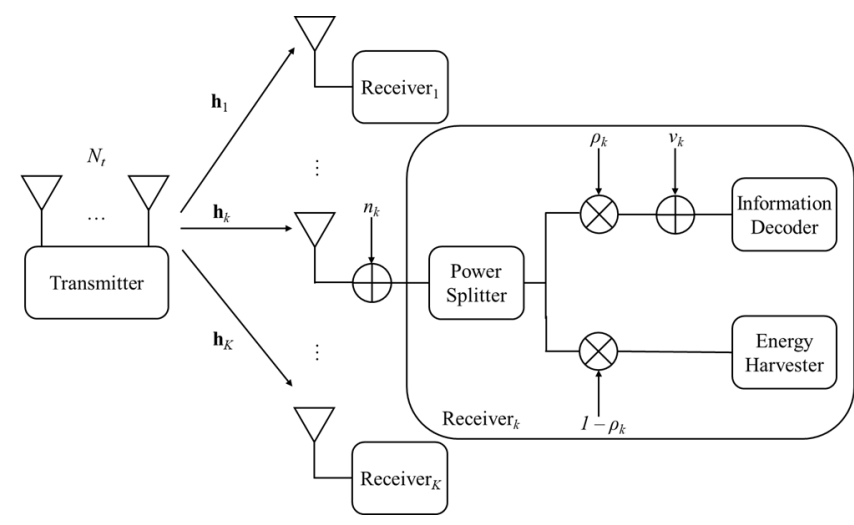

Fig. 1. System setup.

with $\mathcal{M}=\left\{1, \ldots, N_{t}\right\}$ and $\lambda, d=\lambda / 2$, and $\varphi_{k}$ denoting the wavelength, antenna spacing, and angle-of-arrival (AoA), respectively. Finally, the elements of the Rayleigh fading component, $\left[\mathbf{h}_{k}^{\mathrm{NLoS}}\right]_{m}$, represent the fading coefficient of the channel between the $k$-th UE and the $m$-th antenna of the AP. These fading coefficients are independent and identically distributed (i.i.d.) according to $\mathcal{C N}(0,1)$ and they remain constant during a coherence block, but they change independently from one block to the other.

The complex BB received signal at the $k$-th UE is given by

$$
\begin{aligned}
y_{k} & =\mathbf{h}_{k}^{\dagger} \mathbf{x}+n_{k}=\mathbf{h}_{k}^{\dagger} \mathbf{W} \mathbf{s}+n_{k}=\mathbf{h}_{k}^{\dagger} \sum_{i \in \mathcal{K}} \mathbf{w}_{i} s_{i}+n_{k} \\
= & \underbrace{\mathbf{h}_{k}^{\dagger} \mathbf{w}_{k} s_{k}}_{\text {information }}+\underbrace{\mathbf{h}_{k}^{\dagger} \sum_{i \in \mathcal{K} \backslash k} \mathbf{w}_{i} s_{i}}_{\text {inter-user interference }}+n_{k}, \quad k \in \mathcal{K},
\end{aligned}
$$

where $\mathbf{x}=\mathbf{W} \mathbf{s} \in \mathbb{C}^{N_{t}}$ is the transmitted signal, $\mathbf{w}_{k} \in \mathbb{C}^{N_{t}}$ and $s_{k} \sim \mathcal{C N}(0,1)$ correspond to the beamforming $(\mathrm{BF})$ vector and the transmitted symbol for the $k$-th user, respectively, $\mathbf{W}=$ $\left[\begin{array}{lll}\mathbf{w}_{1} & \cdots & \mathbf{w}_{K}\end{array}\right] \in \mathbb{C}^{N_{t} \times K}$ and $\mathbf{s}=\left[\begin{array}{lll}s_{1} & \cdots & s_{K}\end{array}\right]^{T} \in \mathbb{C}^{K}$ represent the precoding matrix and the transmitted symbols vector, respectively, and $n_{k} \sim \mathcal{C N}\left(0, \sigma^{2}\right)$ denotes the receiver's additive white Gaussian noise (AWGN).

Transmission is subject to a number of constraints: (i) the transmit power should not exceed a budget $P_{T}>0$ and (ii) the SINR $\Gamma_{k}$ and harvested power $P_{k}^{h}$ at the $k$-th UE should be at least equal to a minimum target $\bar{\gamma}_{k}>0$ and $\tilde{Q}_{k}>0$, respectively. The harvested power is expressed as a non-linear function of the received power $P_{k}^{r}$, i.e., $P_{k}^{h}=F\left(\left(1-\rho_{k}\right) P_{k}^{r}\right)$. Several models of this function exist in the literature, including the sigmoid function [17], [18] and models based on the characteristic equation of the diode [19], [20]. In general, $F(\cdot)$ is monotonically increasing. Therefore, we can find the inverse mapping $F^{-1}(\cdot)$ and form the equivalent $\mathrm{EH}$ constraint $\left(1-\rho_{k}\right) P_{k}^{r} \geq F^{-1}\left(\tilde{Q}_{k}\right) \triangleq \bar{Q}_{k}$.

2) Problem Formulation: We consider the optimization problem of maximizing the ratios of the received SINRs and harvested powers over their respective target requirements, i.e., $\max \left\{\frac{\Gamma_{1}}{\bar{\gamma}_{1}}, \ldots, \frac{\Gamma_{K}}{\bar{\gamma}_{K}}, \frac{P_{1}^{h}}{\bar{Q}_{1}}, \ldots, \frac{P_{K}^{h}}{\bar{Q}_{K}}\right\}$, subject to the TTP con- straint. To make this SINR / EH balancing problem more tractable, we introduce an auxiliary variable $t$. Thus, the maximization problem is formulated as follows [7]:

$$
\begin{aligned}
& \text { P1: } \max _{\left\{\mathbf{w}_{k}, \rho_{k}, t \forall k \in \mathcal{K}\right\}} t \\
& \text { s.t. } \Gamma_{k}=\frac{\left|\mathbf{h}_{k}^{\dagger} \mathbf{w}_{k}\right|^{2}}{\sum_{i \in \mathcal{K} \backslash k}\left|\mathbf{h}_{k}^{\dagger} \mathbf{w}_{i}\right|^{2}+\sigma^{2}+\frac{\sigma_{c}^{2}}{\rho_{k}}} \geq t \bar{\gamma}_{k} \triangleq \gamma_{k}, \\
& \left(1-\rho_{k}\right) P_{k}^{r}=\left(1-\rho_{k}\right)\left(\sum_{i \in \mathcal{K}}\left|\mathbf{h}_{k}^{\dagger} \mathbf{w}_{i}\right|^{2}+\sigma^{2}\right) \geq t \bar{Q}_{k} \triangleq Q_{k},
\end{aligned}
$$

$0 \leq \rho_{k} \leq 1$,

$\sum_{k \in \mathcal{K}}\left\|\mathbf{w}_{k}\right\|^{2} \leq P_{T}$.

The expressions in Eq. (6b), Eq. (6c), and Eq. (6e) correspond to the QoS, EH, and TTP constraints, respectively. $\sigma_{c}^{2}$ in Eq. (6b) represents the variance of the additive zero-mean complex Gaussian circuit noise $\nu_{k}$ that arises during the BB conversion of the received signal for ID purposes due to phase offsets and non-linearities, as shown in Fig. 1.

It is difficult to solve P1, due to the fact that the QoS and EH constraints are non-convex. Nevertheless, the optimal solution to this optimization problem, which is quasi-convex in $t$, can be obtained by solving the following TTP minimization problem [7]:

$$
\begin{aligned}
& \text { P2: } \min _{\left\{\mathbf{w}_{k}, \rho_{k}\right\}} \sum_{k \in \mathcal{K}}\left\|\mathbf{w}_{k}\right\|^{2} \leq P_{T} \\
& \text { s.t. Eq. (6b) - Eq. (6d). }
\end{aligned}
$$

Notice that $\mathbf{P 2}$ incorporates the variable $t$ in the QoS and $\mathrm{EH}$ constraints. This non-convex problem is solved by using an SDR reformulation. This is achieved by introducing matrix variables $\mathbf{W}_{k} \in \mathbb{C}^{N_{t} \times N_{t}}$ defined as $\mathbf{W}_{k}=\mathbf{w}_{k} \mathbf{w}_{k}^{\dagger}$ [7]:

$$
\begin{aligned}
& \text { P3: } \min _{\left\{\mathbf{W}_{k}, \rho_{k}\right\}} \sum_{k \in \mathcal{K}} \operatorname{tr}\left(\mathbf{W}_{k}\right) \\
& \text { s.t. } \frac{\operatorname{tr}\left(\mathbf{h}_{k} \mathbf{h}_{k}^{\dagger} \mathbf{W}_{k}\right)}{\sum_{j \in \mathcal{K} \backslash k} \operatorname{tr}\left(\mathbf{h}_{k} \mathbf{h}_{k}^{\dagger} \mathbf{W}_{j}\right)+\sigma^{2}+\frac{\sigma_{c}^{2}}{\rho_{k}}} \geq \frac{\gamma_{k}}{1+\gamma_{k}}, k \in \mathcal{K}, \\
& \sum_{j \in \mathcal{K}} \operatorname{tr}\left(\mathbf{h}_{k} \mathbf{h}_{k}^{\dagger} \mathbf{W}_{j}\right)+\sigma^{2} \geq \frac{Q_{k}}{1-\rho_{k}}, k \in \mathcal{K}, \\
& 0 \leq \rho_{k} \leq 1, \mathbf{W}_{k} \succeq 0, \quad k \in \mathcal{K} .
\end{aligned}
$$

The formulation in $\mathbf{P 3}$ is a convex problem and, therefore, can be efficiently solved via numerical software packages such as CVX [21] to obtain $\mathbf{W}_{k}^{\text {opt }}$ and $\rho_{k}^{\text {opt }}(k \in \mathcal{K})$. Moreover, it has been proven that this SDR is tight, i.e., $\operatorname{rank}\left(\mathbf{W}_{k}^{\text {opt }}\right)=1$ [22]. Hence, the optimal BF vectors $\mathbf{w}_{k}^{\text {opt }}$ in $\mathbf{P 2}$ (which are the columns of the optimal fully-digital precoder $\mathbf{W}_{*}$ ) can be recovered from $\mathbf{W}_{k}^{\text {opt }}$ via eigenvalue decomposition. Once $\mathbf{w}_{k}^{\text {opt }}$ and $\rho_{k}^{\text {opt }}$ have been determined, $\mathbf{P 1}$ can be solved for obtaining 


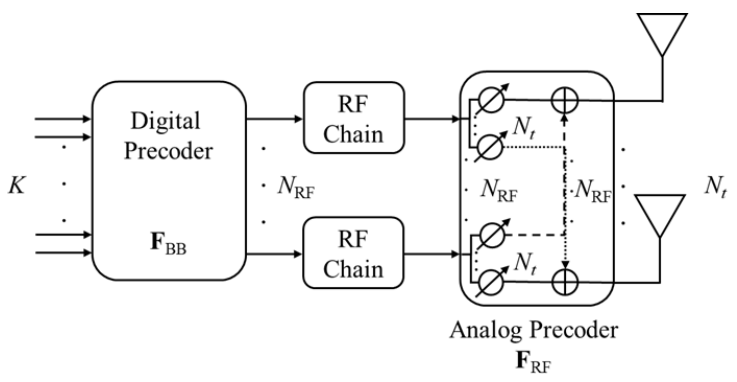

Fig. 2. Analog-digital fully-connected transmitter structure.

$t_{\text {opt }}$ by using the bisection algorithm [7]. Note that $\mathbf{P 3}$ is feasible if and only if $\sum_{k \in \mathcal{K}} \gamma_{k} /\left(1+\gamma_{k}\right) \leq \operatorname{rank}(\mathbf{H})$ [22].

\section{B. Analog-Digital Transmitter}

1) System Model: Next, we consider an A/D transmitter architecture for the AP, wherein $K \leq N_{\mathrm{RF}}<N_{t}$ (i.e., the number of RF chains is smaller than the total number of antennas) and linear precoding is performed on both the analog (RF) and digital (BB) domains, as shown in Fig. 2. Thus, the composite transmitted signal is given by

$$
\mathbf{x}=\mathbf{F} \mathbf{s}=\mathbf{F}_{\mathrm{RF}} \mathbf{F}_{\mathrm{BB}} \mathbf{s}
$$

where $\mathbf{F}_{\mathrm{RF}} \in \mathbb{C}^{N_{t} \times N_{\mathrm{RF}}}$ and $\mathbf{F}_{\mathrm{BB}} \in \mathbb{C}^{N_{\mathrm{RF}} \times K}$ are the RF and BB precoding matrices, respectively, whereas $\mathbf{F} \in \mathbb{C}^{N_{t} \times K}$ denotes the HP matrix given by $\mathbf{F}=\mathbf{F}_{\mathrm{RF}} \mathbf{F}_{\mathrm{BB}}$.

We consider a fully-connected A/D transmitter structure, wherein each RF unit is connected to all antennas through $N_{t}$ phase shifters, as depicted in Fig. 2. This architecture outperforms the sub-array one, where each RF module is connected to a disjoint subset of antennas through several phase shifters, and approaches closely the performance of a fullydigital system when $N_{\mathrm{RF}} \geq 2 K$, in terms of the achieved sumrate, at the cost of higher insertion loss due to the large number of phase shifters and combiners required [8].

Each entry of $\mathbf{F}_{\mathrm{RF}}$ satisfies the constant modulus constraint:

$$
\left[\mathbf{F}_{\mathrm{RF}}\right]_{m, n}=e^{j \phi_{m, n}}, \quad n \in \mathcal{N}, m \in \mathcal{M},
$$

where $\phi_{m, n}$ is the phase of the phase shifter that connects the $m$-th antenna to the $n$-th $\mathrm{RF}$ chain $\left(n \in \mathcal{N}=\left\{1, \ldots, N_{\mathrm{RF}}\right\}\right)$ and $\left|\left[\mathbf{F}_{\mathrm{RF}}\right]_{m, n}\right|=1$. $\mathbf{F}_{\mathrm{BB}}$ can be decomposed as

$$
\mathbf{F}_{\mathrm{BB}}=\left[\begin{array}{lll}
\mathbf{f}_{1}^{\mathrm{BB}} & \cdots & \mathbf{f}_{K}^{\mathrm{BB}}
\end{array}\right],
$$

where $\mathbf{f}_{k}^{\mathrm{BB}} \in \mathbb{C}^{N_{\mathrm{RF}}}$ is the $\mathrm{BB} \mathrm{BF}$ vector for the $k$-th user.

2) Problem Formulation: The joint optimization of $\left\{\mathbf{F}_{\mathrm{RF}}, \mathbf{F}_{\mathrm{BB}}\right\}$ is a challenging task, as mentioned earlier. However, once dealing with the constant modulus constraints, we can compute the RF precoder. Then, given such a constant matrix $\mathbf{F}_{\mathrm{RF}}$, there are two main approaches for computing the $\mathrm{BB}$ precoder. In the first one, we focus on solving the equivalent TTP minimization problem. To this end, we define $\mathbf{F}_{k}^{\mathrm{BB}}=\mathbf{f}_{k}^{\mathrm{BB}}\left(\mathbf{f}_{k}^{\mathrm{BB}}\right)^{\dagger} \in \mathbb{C}^{N_{\mathrm{RF}} \times N_{\mathrm{RF}}}$ and we apply SDR to obtain and solve via CVX the following convex optimization problem, which is the equivalent of $\mathbf{P 3}$ for this scenario:

$$
\begin{aligned}
& \text { P4: } \min _{\left\{\mathbf{F}_{k}^{\mathrm{BB}}, \rho_{k} \forall k \in \mathcal{K}\right\}} \sum_{k \in \mathcal{K}} \operatorname{tr}\left(\mathbf{F}_{\mathrm{RF}} \mathbf{F}_{k}^{\mathrm{BB}} \mathbf{F}_{\mathrm{RF}}^{\dagger}\right) \\
& \text { s.t. } \frac{\operatorname{tr}\left(\mathbf{h}_{k} \mathbf{h}_{k}^{\dagger} \mathbf{F}_{\mathrm{RF}} \mathbf{F}_{k}^{\mathrm{BB}} \mathbf{F}_{\mathrm{RF}}^{\dagger}\right)}{\sum_{j \in \mathcal{K} \backslash k} \operatorname{tr}\left(\mathbf{h}_{k} \mathbf{h}_{k}^{\dagger} \mathbf{F}_{\mathrm{RF}} \mathbf{F}_{j}^{\mathrm{BB}} \mathbf{F}_{\mathrm{RF}}^{\dagger}\right)+\sigma^{2}+\frac{\sigma_{c}^{2}}{\rho_{k}}} \geq \frac{\gamma_{k}}{1+\gamma_{k}}, \\
& \sum_{j \in \mathcal{K}} \operatorname{tr}\left(\mathbf{h}_{k} \mathbf{h}_{k}^{\dagger} \mathbf{F}_{\mathrm{RF}} \mathbf{F}_{j}^{\mathrm{BB}} \mathbf{F}_{\mathrm{RF}}^{\dagger}\right)+\sigma^{2} \geq \frac{Q_{k}}{1-\rho_{k}}, \\
& 0 \leq \rho_{k} \leq 1, \mathbf{W}_{k} \succeq 0 .
\end{aligned}
$$

Alternatively, we can seek to minimize the Euclidean distance between the optimal fully-digital precoder $\mathbf{W}_{*}$ derived in Sec. II-A and the hybrid one:

$$
\text { P5: } \min _{\left\{\mathbf{F}_{\mathrm{BB}}\right\}}\left\|\mathbf{W}_{*}-\mathbf{F}_{\mathrm{RF}} \mathbf{F}_{\mathrm{BB}}\right\|_{F}^{2} \text { s.t. } \mathbf{F}_{\mathrm{RF}} \in \mathcal{F} \text {. }
$$

In Eq. (13), $\mathcal{F}$ denotes the set of matrices whose entries satisfy the constant modulus constraint. This problem leads to the wellknown least squares (LS) solution [23], i.e.,

$$
\mathbf{F}_{\mathrm{BB}}=\mathbf{F}_{\mathrm{RF}}^{\#} \mathbf{W}_{*}=\mathbf{F}_{\mathrm{RF}}^{\dagger}\left(\mathbf{F}_{\mathrm{RF}} \mathbf{F}_{\mathrm{RF}}^{\dagger}\right)^{-1} \mathbf{W}_{*} .
$$

\section{Proposed Hybrid Precoding Schemes}

\section{A. Random and SVD-based Phase Selection}

In random phase selection (RPS), the phases $\phi_{m, n}$ in Eq. (10) are randomly selected from $\mathcal{U}[-\pi, \pi]$. In SVD-based phase selection (SPS), on the other hand, we consider the SVD of the channel matrix $\mathbf{H}=\left[\begin{array}{lll}\mathbf{h}_{1} & \cdots & \mathbf{h}_{K}\end{array}\right]^{T} \in \mathbb{C}^{K \times N_{t}}$ given by $\mathbf{H}=\mathbf{U} \boldsymbol{\Lambda} \mathbf{V}^{\dagger}$, where the columns of the unitary matrices $\mathbf{U}$ and $\mathbf{V}=\left[\begin{array}{lll}\mathbf{v}_{1} & \cdots & \mathbf{v}_{N_{t}}\end{array}\right]$ are the left and right singular vectors of $\mathbf{H}$, respectively, and $\boldsymbol{\Lambda}$ is a diagonal matrix that holds on its diagonal the singular values of $\mathbf{H}$ in a descending order. In this case, we define the phases of the RF precoder as [16]

$$
\phi_{m, n}=\theta_{m, n}, \quad m \in \mathcal{M}, n \in \mathcal{N},
$$

where $\theta_{m, n}$ is the phase of the $m$-th element of $\mathbf{v}_{n}$. Once fixing the RF precoder via RPS or SPS, the BB precoder can be obtained by solving P4 (first approach mentioned in Sec. II-B).

\section{B. Stochastic Optimization Approach}

The non-convex joint optimization problem can be solved iteratively based on $\mathbf{P 5}$ (second approach mentioned in Sec. II-B). Let the RF precoder be expressed as $\mathbf{F}_{\mathrm{RF}}=g(\boldsymbol{\Phi})=$ $e^{j \boldsymbol{\Phi}}$, where $\boldsymbol{\Phi} \in \mathbb{R}^{N_{t} \times N_{\mathrm{RF}}}$. Then, the cost function of P5 is given by $f(\boldsymbol{\Phi})=\left\|\mathbf{W}_{*}-g(\boldsymbol{\Phi}) \mathbf{F}_{\mathrm{BB}}^{(i)}\right\|_{F}^{2}$. We start from a random RF precoder $\mathbf{F}_{\mathrm{RF}}^{(0)}$, where the phases $\boldsymbol{\Phi}_{0}$ are computed according to the HP-RPS scheme. At the $i$-th iteration, given $\mathbf{F}_{\mathrm{RF}}^{(i)}$, the BB precoding matrix is computed by

$$
\mathbf{F}_{\mathrm{BB}}^{(i)}=\left(\mathbf{F}_{\mathrm{RF}}^{(i)}\right)^{\dagger}\left(\mathbf{F}_{\mathrm{RF}}^{(i)}\left(\mathbf{F}_{\mathrm{RF}}^{(i)}\right)^{\dagger}\right)^{-1} \mathbf{W}_{*}, i=0,1, \ldots
$$


For the update of the RF precoding matrix, we have to find $\boldsymbol{\Phi}_{i+1}$ that minimizes the cost function $f(\boldsymbol{\Phi})$ :

$$
\mathbf{F}_{\mathrm{RF}}^{(i+1)}=\underset{\boldsymbol{\Phi}}{\operatorname{argmin}}\left\|\mathbf{W}_{*}-g(\boldsymbol{\Phi}) \mathbf{F}_{\mathrm{BB}}^{(i)}\right\|_{F}^{2} .
$$

We note that $f$ has multiple local minima. Nevertheless, this function is smooth and its gradient is given in Eq. (19) at the top of the next page [23].

Consider a random matrix $\mathbf{S} \sim \mathcal{M N}_{N_{t} \times N_{\mathrm{RF}}}(\mathbf{M}, \boldsymbol{\Sigma}, \mathbf{\Psi})$ with $\operatorname{vec}(\mathbf{S}) \sim \mathcal{N}_{N_{t} N_{\mathrm{RF}}}(\operatorname{vec}(\mathbf{M}), \boldsymbol{\Psi} \otimes \boldsymbol{\Sigma})$. Let $\mathbf{M}=\mathbf{0}_{N_{t} \times N_{\mathrm{RF}}}$, $\boldsymbol{\Sigma}=b^{2} \mathbf{I}_{N_{t}}, \mathbf{\Psi}=c^{2} \mathbf{I}_{N_{\mathrm{RF}}}$, and $\mu=b c$. Then, the probability density function (p.d.f.) of this matrix is given by

$$
p(\mathbf{S}, \mu)=\frac{e^{-\frac{1}{2 \mu^{2}}\|\mathbf{S}\|_{F}^{2}}}{\mu^{N_{t} \times N_{\mathrm{RF}}} \sqrt{(2 \pi)^{N_{t} \times N_{\mathrm{RF}}}}} .
$$

We apply convolutional smoothing to the cost function $f$ by using the weighting Gaussian p.d.f. $p(\mathbf{S}, \mu)$. The smoothed approximation of the cost function can be expressed as:

$$
\begin{aligned}
f_{\mu}(\boldsymbol{\Phi}) & =(p * f)(\boldsymbol{\Phi})=\int_{\mathbb{R}^{N_{t} \times N_{\mathrm{RF}}}} p(\mathbf{S}, \mu) f(\mathbf{\Phi}-\mathbf{S}) d \mathbf{S} \\
& =\int_{\mathbb{R}^{N_{t} \times N_{\mathrm{RF}}}} p(\mathbf{S}) f(\mathbf{\Phi}-\mu \mathbf{S}) d \mathbf{S},
\end{aligned}
$$

where $p(\mathbf{S})=p(\mathbf{S}, 1)$. Hence, $f_{\mu}(\boldsymbol{\Phi})=\mathbb{E}_{\mathbf{S}}[f(\boldsymbol{\Phi}-\mu \mathbf{S})]$ and, therefore,

$$
\nabla_{\boldsymbol{\Phi}} f_{\mu}(\boldsymbol{\Phi})=\mathbb{E}_{\mathbf{S}}\left[\nabla_{\boldsymbol{\Phi}} f(\boldsymbol{\Phi}-\mu \mathbf{S})\right] .
$$

The two-sided gradient estimate is given by Eq. (23) at the top of the next page [23]. In Eq. (23), $N$ denotes the number of samples used for the gradient estimation; $N=1$ corresponds to a stochastic approach.

Based on the above, instead of trying to minimize the original cost function, we attempt to solve the following stochastic optimization task in each iteration:

$$
\text { P6: } \min _{\mathbf{\Phi}}\left\{f_{\mu_{i}}(\boldsymbol{\Phi})=\mathbb{E}_{\mathbf{S}}\left[f\left(\mathbf{\Phi}-\mu_{i} \mathbf{S}\right)\right]\right\}
$$

where the sequence $\left\{\mu_{i}\right\}_{i \in \mathbb{N}}$ is strictly decreasing with $\lim _{i \rightarrow \infty} \mu_{i}=0$. In practice, a sequence with finite length $L$ is sufficient for the approximation. The SGD algorithm is employed for the update of the phases in each iteration. The HP via stochastic approximation with Gaussian smoothing (HPSAGS) and SGD algorithms are presented in Algorithm 1 and Algorithm 2, respectively, where $\epsilon_{t}$ and $\epsilon$ are the convergence accuracy and threshold of SGD, while $\eta$ is its step size.

\section{Computational Complexity and Convergence}

The computational complexity of the interior-point algorithm that is used by CVX to solve the dual problem of an $X$-dimensional optimization task with $Y$ variables is $\mathcal{O}\left(\sqrt{Y X}\left(Y^{3} X^{2}+Y^{2} X^{3}\right)\right)$ [7]. Regarding P3, we have $X=$ $N_{t}$ and $Y=K$ when a fully-digital transmitter is utilized, whereas $X=N_{\mathrm{RF}}$ and $Y=K$ when HP-RPS or HP-SPS is applied. In any case, the algorithm is guaranteed to converge to the optimal solution, since the SDR is tight.
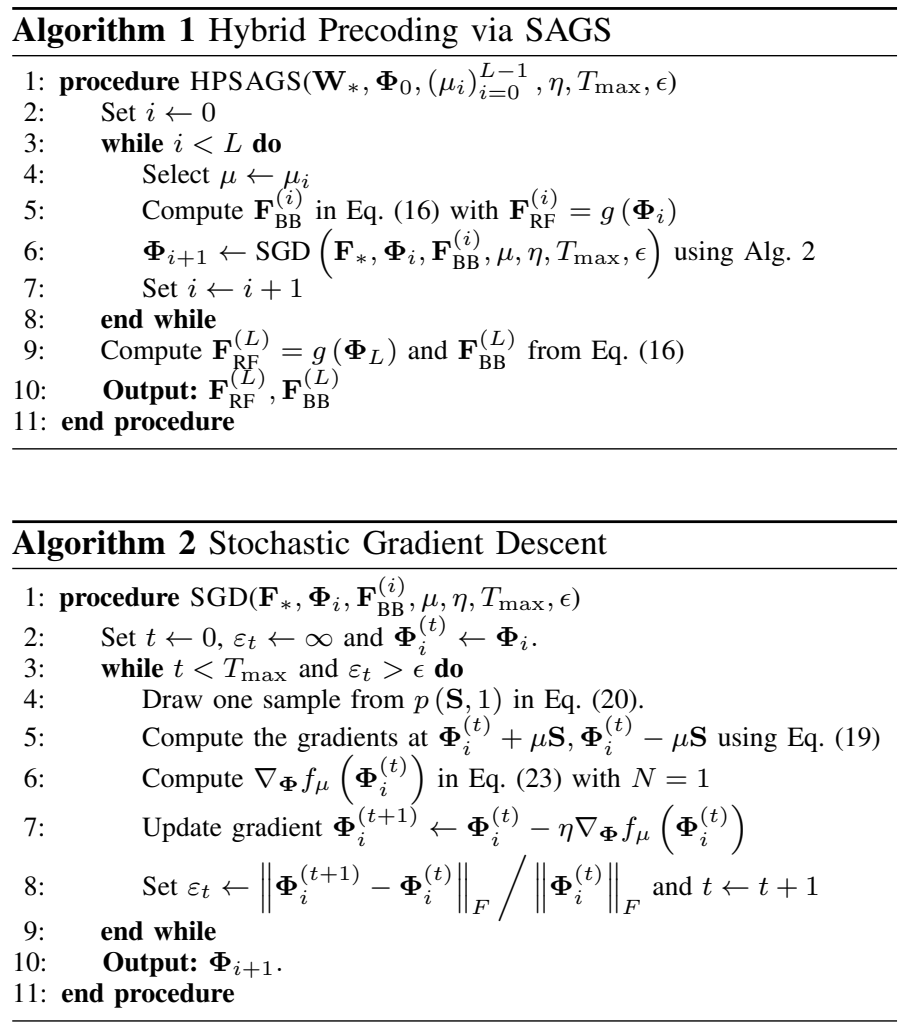

The computational efficiency of the HP-SAGS algorithm, on the other hand, which runs much faster than, for instance, MDP [23], is expressed in terms of its worst-case complexity $\mathcal{O}\left(N_{t} N_{\mathrm{RF}}^{2} L T_{\max }\right)$, where $T_{\max }$ represents the number of iterations [23]. Moreover, the convergence of this algorithm cannot be proven, since the optimality of alternating minimization approaches for non-convex optimization tasks is still an open problem [24]. Nonetheless, we validated via numerical simulations that the relative error is strictly decreasing in the vast majority of the independent Monte Carlo simulation runs.

\section{Total Power Consumption at the Transmitter}

Based on [25], [26], the total power consumption at the AP for the cases where a fully-digital or an A/D fully-connected transmitter architecture is utilized is given by

$$
\begin{aligned}
P_{D}= & N_{t}\left(N_{t}+1\right)\left(\frac{1}{\eta_{0}} P_{T X}\right) \\
& +N_{t}\left(P_{R F C}+P_{D A C}\right)+P_{B B} \\
P_{A / D}= & N_{t}\left(N_{\mathrm{RF}}+1\right) \frac{1}{\eta_{0}} P_{T X}+N_{\mathrm{RF}}\left(P_{R F C}+P_{D A C}\right) \\
& +P_{B B}+N_{t} N_{\mathrm{RF}} P_{P S}
\end{aligned}
$$

where $0 \leq \eta_{0} \leq 1$ is the power amplifier efficiency, $P_{T X}$ denotes the transmission power, $P_{B B}$ corresponds to the power consumption of the $\mathrm{BB}$ unit, and $P_{R F C}, P_{D A C}$, and $P_{P S}$ refer to the power consumption of each RF chain, digital-to-analog converter, and phase shifter, respectively. The power consumption reduction that can be achieved with the $\mathrm{A} / \mathrm{D}$ transmitter architecture is given by $\eta_{p c}=P_{A / D} / P_{D}$. Clearly, smaller values of $\eta_{p c}$ imply larger power consumption reduction. 


$$
\nabla_{\boldsymbol{\Phi}} f(\boldsymbol{\Phi})=-2 \operatorname{Re}\left\{j g(\boldsymbol{\Phi}) \odot\left(\mathbf{W}_{*}-g(\boldsymbol{\Phi}) \mathbf{F}_{\mathrm{BB}}^{(i)}\right)^{*}\left(\mathbf{F}_{\mathrm{BB}}^{(i)}\right)^{T}\right\}
$$

$$
\nabla_{\boldsymbol{\Phi}} f_{\mu}(\boldsymbol{\Phi})=\frac{1}{2 N} \sum_{n=1}^{N}\left(\nabla_{\boldsymbol{\Phi}} f\left(\boldsymbol{\Phi}+\mu \mathbf{S}_{[n]}\right)+\nabla_{\boldsymbol{\Phi}} f\left(\boldsymbol{\Phi}-\mu \mathbf{S}_{[n]}\right)\right)
$$

\section{Fixed Hybrid Precoding Schemes}

Consider a HP method where the RF precoder $\mathbf{F}_{\mathrm{RF}} \in$ $\mathbb{C}^{N_{t} \times N_{\mathrm{RF}}}$ is computed based on the HP-RPS or HP-SPS schemes. Let us define the effective channel after the application of the RF precoder $\tilde{\mathbf{H}}=\left[\begin{array}{lll}\tilde{\mathbf{h}}_{1} & \cdots & \tilde{\mathbf{h}}_{K}\end{array}\right]^{T} \in \mathbb{C}^{K \times N_{\mathrm{RF}}}$ as $\tilde{\mathbf{H}}=\mathbf{H F}_{\mathrm{RF}}$, where $\tilde{\mathbf{h}}_{k} \in \mathbb{C}^{N_{\mathrm{RF}}}$. In HP-RPS-ZF or HP-SPS$\mathrm{ZF}$, a ZF BB precoder $\mathbf{F}_{\mathrm{BB}}^{\mathrm{ZF}} \in \mathbb{C}^{N_{\mathrm{RF}} \times K}$ follows the RPS or SPS based RF precoder, respectively, in order to manage the inter-user interference. This precoder is defined as

$$
\mathbf{F}_{\mathrm{BB}}^{\mathrm{ZF}}=\tilde{\mathbf{H}}^{\#}=\mathbf{F}_{\mathrm{RF}}^{\dagger} \mathbf{H}^{\dagger}\left(\mathbf{H} \mathbf{F}_{\mathrm{RF}} \mathbf{F}_{\mathrm{RF}}^{\dagger} \mathbf{H}^{\dagger}\right)^{-1} .
$$

We express the BB precoder in terms of its BF vectors according to Eq. (11). Then, we decompose these non-normalized $\mathrm{BF}$ vectors as $\mathbf{f}_{k}^{\mathrm{BB}}=\sqrt{P_{k}} \mathbf{f}_{k}^{\mathrm{BB}, \mathrm{f}}(k \in \mathcal{K})$, where $\mathbf{f}_{k}^{\mathrm{BB}, \mathrm{f}}$ is the normalized $\mathrm{BB} \mathrm{BF}$ vector for the $k$-th user with $\left\|\mathbf{f}_{k}^{\mathrm{BB}, \mathrm{f}}\right\|=1$ and $P_{k}$ is the power allocated to the $k$-th UE. Let $G_{k j}=\left|\tilde{\mathbf{h}}_{k}^{\dagger} \mathbf{f}_{j}^{\mathrm{BB}, \mathrm{f}}\right|^{2}$. Then, in order to obtain the optimal power allocation and PS parameters, we formulate the following TTP minimization problem:

$$
\begin{aligned}
& \text { P7: } \min _{\left\{P_{k}, \rho_{k}\right\}} \sum_{k \in \mathcal{K}} P_{k} \\
& \text { s.t. }\left(\frac{1}{\gamma_{k}}+1\right) G_{k k} P_{k} \geq \frac{1}{\rho_{k}} \sigma_{c}^{2}+\sum_{j \in \mathcal{K}} G_{k j} P_{j}+\sigma^{2}, \quad k \in \mathcal{K}
\end{aligned}
$$$$
\sum_{j \in \mathcal{K}} G_{k j} P_{j}+\sigma^{2} \geq \frac{Q_{k}}{1-\rho_{k}}, \quad k \in \mathcal{K}
$$

$$
P_{k} \geq 0,0 \leq \rho_{k} \leq 1, \quad k \in \mathcal{K}
$$

This problem is convex and can be solved using CVX.

\section{Performance Evaluation via Simulations}

In this section, we comparatively evaluate the performance of the proposed HP methods versus the one achieved by the optimal fully-digital precoding scheme via numerical simulations. The simulation parameters are summarized in Table I. Note that, for convenience and without loss of generality, we consider the same SINR and EH thresholds for all users.

In Fig. 3 is illustrated the minimum achievable SINR and $\mathrm{EH}$ ratio for an $\mathrm{EH}$ threshold ranging in $-25 \mathrm{dBm} \leq \bar{Q} \leq$ $-5 \mathrm{dBm}$. We see that the HP-SAGS outperforms the other methods and provides a favorable complexity-performance trade-off. We also note that the variants that employ a ZF BB precoder perform worse than their counterparts. The reason
TABLE I

Simulation PARAMETERS

\begin{tabular}{|l|l|}
\hline Number of transmit antennas & $N_{t}=8$ \\
\hline Number of RF chains (A/D transmitter) & $N_{\mathrm{RF}}=4$ \\
\hline Number of single-antenna terminals & $K=2$ \\
\hline Distance of the $k$-th user from the AP [m] & $d_{k} \sim \mathcal{U}(1,5)$ \\
\hline AoA for the $k$-th user [rad] & $\varphi_{k} \sim \mathcal{U}(-\pi, \pi)$ \\
\hline Carrier frequency [MHz] & 915 \\
\hline Rician factor [dB] & 5 \\
\hline Reference distance [m] & 1 \\
\hline Path loss at reference distance [dB] & 31.67 \\
\hline Path loss exponent & 2.5 \\
\hline AWGN power [dBm] & -70 \\
\hline Circuit noise power [dBm] & -50 \\
\hline Transmit power budget [W] & 2 \\
\hline SINR threshold [dB] & $\bar{\gamma}_{k}=\bar{\gamma}=10$ \\
\hline EH threshold [dBm] & $Q_{k}=Q=-15$ \\
\hline Power amplifier efficiency & $\eta_{0}=0.5$ \\
\hline BB unit power consumption [mW] & $P_{B B}=5$ \\
\hline RF chain power consumption [mW] & $P_{R F C}=40$ \\
\hline DAC power consumption [mW] & $P_{D A C}=200$ \\
\hline Phase shifter power consumption [mW] & $P_{P S}=30$ \\
\hline
\end{tabular}

for that is twofold: (i) In this case, the digital precoder is not optimized. In fact, interference cancellation improves the SINR but reduces the harvested power at the terminals. (ii) $\mathrm{ZF}$ precoding is inherently power-inefficient. Furthermore, we notice that for more stringent EH constraints, the performance gap between the fully-digital and HP solutions is reduced.

In Fig. 4, the power consumption reduction achieved by HPSAGS and HP-SPS as a function of the number of RF chains in the A/D transmitter is depicted, assuming $N_{t}=8$. We observe that HP-SAGS provides greater power consumption reduction than HP-SPS. This is essentially translated into smaller transmission power, since the remaining components of the power consumption equations are the same. Fig. 4 indicates also that as the number of RF chains increases, the power saving attributed to HP is reduced, as expected.

\section{CONCLUSIONS}

In this paper, we presented several frequency-agnostic lowcomplexity HP methods for broadcast MISO SWIPT, as a step towards end-to-end energy-sustainable wireless communication networks. Numerical simulations demonstrated that the proposed HP-SAGS scheme achieves good performance and substantial power consumption reduction without suffering from the inherent computational complexity of other approaches such as the ones described in [10]-[12]. 


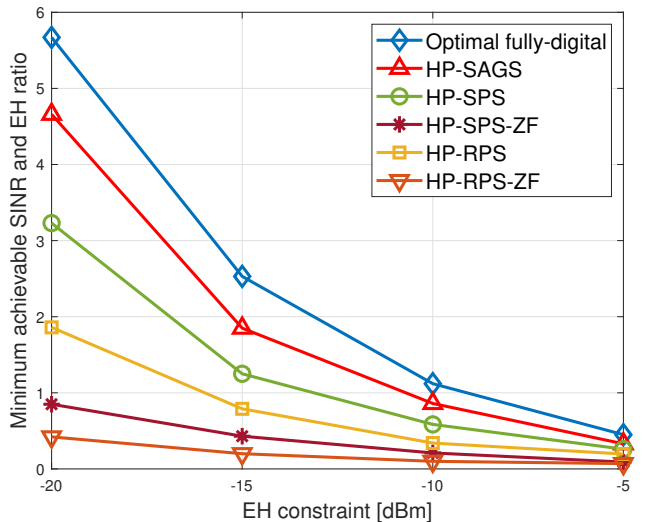

Fig. 3. Minimum achievable SINR and EH ratio as a function of the EH threshold for fixed SINR threshold $\bar{\gamma}=10 \mathrm{~dB}$

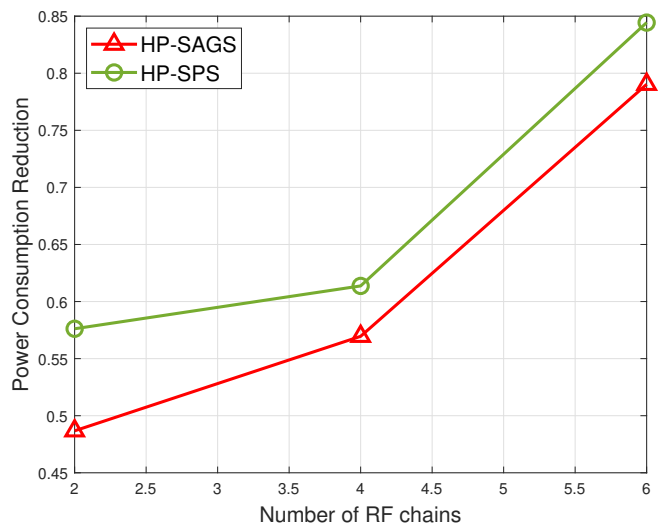

Fig. 4. Power consumption reduction factor as a function of the number of RF chains assuming $N_{t}=8$ antennas.

\section{ACKNOWLEDGMENT}

This work has been funded from the H2020 ERC APOLLO (Grant agreement No. 819819), Republic of Cyprus RIF IRIDA (INFRASTRUCTURES/1216/0017), and UK EPSRC MANGO (EP/P009670/1).

\section{REFERENCES}

[1] Ericsson, "Mobility Report," Nov. 2019.

[2] I. Krikidis, S. Timotheou, S. Nikolaou, G. Zheng, D. W. K. Ng, and R. Schober, "Simultaneous Wireless Information and Power Transfer in Modern Communication Systems," IEEE Commun. Mag., vol. 52, no. 11, pp. 104-110, Nov. 2014.

[3] S. Timotheou, I. Krikidis, G. Zheng, and B. Ottersten, "Beamforming for MISO Interference Channels with QoS and RF Energy Transfer," IEEE Trans. Wireless Commun., vol. 13, no. 5, pp. 2646-2658, May 2014.

[4] S. Timotheou, I. Krikidis, S. Karachontzitis, and K. Berberidis, "Spatial Domain Simultaneous Information and Power Transfer for MIMO Channels," IEEE Trans. Wireless Commun.,, vol. 14, no. 8, pp. 4115-4128, Aug. 2015.

[5] J. Rubio, A. Pascual-Iserte, D. P. Palomar, and A. Goldsmith, "Joint Optimization of Power and Data Transfer in Multiuser MIMO Systems," IEEE Trans. Signal Process., vol. 65, no. 1, pp. 212-227, Jan. 2017.

[6] Q. Li and J. Lin, "Optimal SWIPT Beamforming for MISO Interfering Broadcast Channels with Multi - Type Receivers," in 26th Eur. Signal Process. Conf. (EUSIPCO), Rome, Italy, 3-7 Sept., 2018.
[7] J. Zhang, G. Zheng, I. Krikidis, and R. Zhang, "Specific Absorption RateAware Beamforming in MISO Downlink SWIPT Systems," IEEE Trans. Commun., Nov. 2019, (accepted for publication).

[8] A. F. Molisch, V. V. Ratnam, S. Han, Z. Li, S. L. H. Nguyen, L. Li, and K. Haneda, "Hybrid Beamforming for Massive MIMO: A Survey," IEEE Commun. Mag., vol. 55, no. 9, pp. 134-141, Sep. 2017.

[9] O. E. Ayach, S. Rajagopal, S. Abu-Surra, Z. Pi, and R. W. Heath, "Spatially Sparse Precoding in Millimeter Wave MIMO Systems," IEEE Trans. Wireless Commun., vol. 13, no. 3, pp. 1499-1513, Mar. 2014.

[10] W. Ni, X. Dong, and W.-S. Lu, "Near-Optimal Hybrid Processing for Massive MIMO Systems via Matrix Decomposition," IEEE Trans. Signal Process., vol. 65, no. 15, pp. 3922-3933, Aug. 2017.

[11] X. Yu, J. C. Shen, J. Zhang, and K. B. Letaief, "Hybrid Precoding Design in Millimeter Wave MIMO Systems: An Alternating Minimization Approach," in IEEE Global Commun. Conf. (GLOBECOM), San Diego, CA, USA, 2015, 6-10 Dec.

[12] C.-E. Chen, "An Iterative Hybrid Transceiver Design Algorithm for Millimeter Wave MIMO Systems," IEEE Wireless Commun. Letters, vol. 4, no. 3, pp. 285-288, Jun. 2015.

[13] O. T. Demir and T. E. Tuncer, "Antenna Selection and Hybrid Beamforming for Simultaneous Wireless Information and Power Transfer in MultiGroup Multicasting Systems," IEEE Trans. Wireless Commun., vol. 15, no. 10, pp. 6948-6962, Oct. 2016.

[14] L. Dai, B. Wang, M. Peng, and S. Chen, "Hybrid Precoding-Based Millimeter-Wave Massive MIMO-NOMA With Simultaneous Wireless Information and Power Transfer," IEEE J. Sel. Areas Commun., vol. 37 no. 1, pp. 131-141, Jan. 2019.

[15] W. Hao, G. Sun, F. Zhou, D. Mi, J. Shi, P. Xiao, and V. C. M. Leung "Energy-Efficient Hybrid Precoding Design for Integrated MulticastUnicast Millimeter Wave Communications With SWIPT," IEEE Trans. Veh. Technol., vol. 68, no. 11, pp. 10956-10968, Nov. 2019.

[16] A. Li and C. Masouros, "Energy-Efficient SWIPT: From Fully Digital to Hybrid Analog-Digital Beamforming," IEEE Trans. Veh. Technol., vol. 67, no. 4, pp. 3390-3405, Apr. 2018.

[17] E. Boshkovska, D. W. K. Ng, N. Zlatanov, and R. Schober, "Practical Non-linear Energy Harvesting Model and Resource Allocation for SWIPT Systems," IEEE Commun. Lett., vol. 19, no. 12, pp. 2082-2085, Dec. 2015.

[18] S. Wang, M. Xia, K. Huang, and Y.-C. Wu, "Wirelessly Powered TwoWay Communication With Nonlinear Energy Harvesting Model: Rate Regions Under Fixed and Mobile Relay," IEEE Trans. Wireless Commun., vol. 16, no. 12, pp. 8190-8204, Dec. 2017.

[19] B. Clerckx and E. Bayguzin, "Waveform Design for Wireless Power Transfer," IEEE Trans. Signal Process, vol. 64, no. 23, pp. 6313-6328, Dec. 2016.

[20] M. R. Vedady Moghada, Y. Zeng, and R. Zhang, "Waveform Optimization for Radio-Frequency Wireless Power Transfer," in 18th Int. Workshop Signal Process. Adv. Wireless Commun. (SPAWC), Sapporo, Japan, 3-6 July, 2017

[21] M. Grant and S. Boyd. (2020, Jan.) CVX: Matlab Software for Disciplined Convex Programming. Version 2.2. [Online]. Available: http://cvxr.com/cvx/

[22] Q. Shi, L. Liu, W. Xu, and R. Zhang, "Joint Transmit Beamforming and Receive Power Splitting for MISO SWIPT Systems," IEEE Trans. Wireless Commun., vol. 13, no. 6, pp. 3269-3280, Jun. 2014.

[23] G. K. Papageorgiou, M. Sellathurai, K. Ntougias, and C. B. Papadias, "A Stochastic Optimization Approach to Hybrid Processing in Massive MIMO Systems," IEEE Wireless Commun. Letters, vol. 9, no. 6, pp. 770773, Jun. 2020.

[24] A. Patrascu and I. Necoara, "Efficient Random Coordinate Descent A1gorithms for Large-Scale Structured Nonconvex Optimization," J. Global Optim., vol. 61, no. 1, pp. 19-46, 2015.

[25] R. Mendez-Rial, C. Rusu, N. Gonzalez-Prelcic, A. Alkhateeb, and R. W. J. Heath, "Hybrid MIMO Architectures for Millimeter Wave Communications: Phase Shifters or Swithes?" IEEE Access, vol. 4, pp. 247-267, Jan. 2016

[26] C. Masouros, M. Sellathurai, and T. Ratnarajah, "Maximizing Energy Efficiency in the Vector Precoded MU-MISO Downlink by Selective Perturbation," IEEE Trans. Wireless Commun., vol. 13, no. 9, pp. 4974 4984, Sep. 2014 